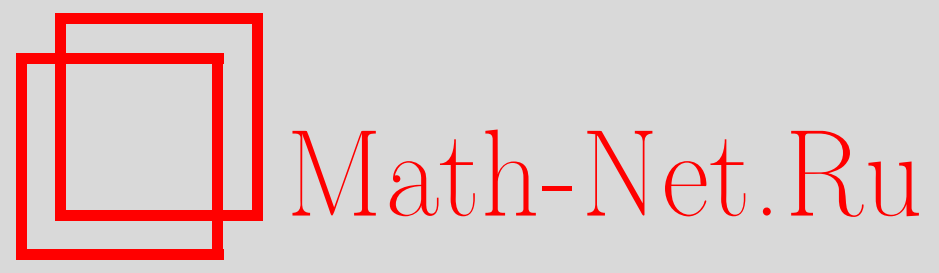

О. В. Кузьмин, О. В. Леонова, Полиномы Тушара и их приложения, Дискрет. матем., 2000, том 12, выпуск 3, 6071

DOI: https://doi.org/10.4213/dm343

Использование Общероссийского математического портала Math-Net.Ru подразумевает, что вы прочитали и согласны с пользовательским соглашением http://www . mathnet.ru/rus/agreement

Параметры загрузки:

IP: 54.198 .64 .247

26 апреля 2023 г., 11:13:45

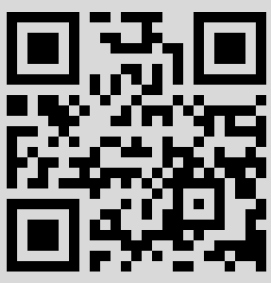


УДК 519.1

\title{
Полиномы Тушара и их приложения
}

\author{
(C) 2000 г. О. В. Кузьмин, О. В. Леонова
}

Рассматриваемые в статье полиномы являются одним из обобщений полиномов Белла и введены Тушаром в связи с изучением некоторых циклических подстановок. Для полиномов Тушара получены дифференциально-разностные уравнения и рекуррентные соотношения, а также обобщения некоторых известных ранее свойств полиномов, их перечислительные интерпретации и вероятностные приложения, в частности, при описании одноканальной системы массового обслуживания.

\section{1. Введение}

Понятие полинома разбиений, полинома от нескольких переменных, определяемого с помощью суммы по различным разбиениям значений его индекса, введено Беллом в [1]. Один из таких полиномов, связанный с производными от композиции функций, в книге [2] назван полиномом Белла. Ряд свойств коэффициентов $n$-го полинома Белла, так называемых частичных полиномов Белла $A_{n, k}$, и сопряженных с ними полиномов Платонова $B_{n, k}$ приведен в статье [3].

Тушар (см. [4]) в связи с изучением некоторых циклических подстановок ввел ряд обобщений полиномов Белла. Для одного из таких обобщений $T_{n, k}$, названного полиномами Тушара, в [5] получены экспоненциальные производящие функции и рекуррентные соотношения. В [6] рассматриваются свойства некоторых частных случаев полиномов $T_{n, k}$ и вновь введенных полиномов Тушара $C_{n, k}$. Полученные формулы использованы при нахождении некоторых вероятностей в задаче о случайных блужданиях.

В данной статье продолжается начатое авторами в [7] изучение полиномов Тушара, при этом получены обобщения некоторых из приведенных в [3] свойств полиномов $A_{n, k}$, перечислительные интерпретации и вероятностные приложения. Во втором разделе приводятся необходимые определения и свойства. В третьем разделе для полиномов $T_{n, k}$ получены дифференциально-разностные уравнения и рекуррентные соотношения, которые позволяют по фиксированной $i$-й строке матрицы, составленной из этих полиномов, восстановить все ее начальные строки. Рассматривается ряд частных случаев. В четвертом разделе статьи получено новое соотношение для полиномов $C_{n, k}$. Известные и новые перечислительные интерпретации изучаемых полиномов Тушара обсуждаются в пятой части. В последней части приводится пример приложения полиномов Тушара при описании одноканальной системы массового обслуживания. 


\section{2. Основные понятия и вспомогательные результаты}

Полиномы Тушара $T_{n, k}=T_{n, k}(x, y)$ для $n, k \geqslant 1, k \leqslant n$, имеют вид

$$
T_{n, k}(x, y)=\sum \frac{n !}{k_{1} ! \ldots k_{n} ! r_{1} ! \ldots r_{n} !}\left(\frac{x_{1}}{1 !}\right)^{k_{1}} \ldots\left(\frac{x_{n}}{n !}\right)^{k_{n}}\left(\frac{y_{1}}{1 !}\right)^{r_{1}} \ldots\left(\frac{y_{n}}{n !}\right)^{r_{n}}
$$

(см. [5]), где $x=\left(x_{1}, x_{2}, \ldots\right), y=\left(y_{1}, y_{2}, \ldots\right)$ - формальные переменные, а суммирование ведется по всем таким наборам $\left(k_{1}, \ldots, k_{n} ; r_{1}, \ldots, r_{n}\right)$ целых неотрицательных чисел, что

$$
\sum_{i=1}^{n} k_{i}=k, \quad \sum_{i=1}^{n} i\left(k_{i}+r_{i}\right)=n .
$$

Дополнительно полагают $T_{0,0}(x, y)=1$.

Из (1) следует, что

$$
T_{n, 0}(x, y)=Y_{n}(y)
$$

где $Y_{n}(y)=Y_{n}\left(y_{1}, \ldots, y_{n}\right)-$ хорошо известные полиномы Белла (см., например, [2]) и

$$
T_{n, n}(x, y)=x_{1}^{n}=A_{n, n}(x),
$$

здесь $A_{n, k}(x)=A\left(n, k ; x_{1}, \ldots, x_{n-k+1}\right)$ - частичные полиномы Белла (см., например, [8]). Известна производящая функция

$$
T_{k}(x, y ; t)=\sum_{n=k}^{\infty} T_{n k}(x, y) \frac{t^{n}}{n !}=(x(t))^{k} \frac{e^{y(t)}}{k !}
$$

где

$$
x(t)=\sum_{i=1}^{\infty} x_{i} \frac{t^{i}}{i !}, \quad y(t)=\sum_{i=1}^{\infty} y_{i} \frac{t^{i}}{i !} .
$$

Полиномы Тушара и Белла связаны соотношением

$$
\sum_{k=0}^{n} T_{n, k}(x, y)=\sum_{j=0}^{n}\left(\begin{array}{l}
n \\
j
\end{array}\right) Y_{j}(x) Y_{n-j}(y)
$$

\section{3. Рекуррентные соотношения для полиномов Тушара}

Выведем несколько рекуррентных соотношений для полиномов Тушара $T_{n k}(x, y)$.

Пусть, по-прежнему, $x=\left(x_{1}, x_{2}, \ldots\right)$ и $y=\left(y_{1}, y_{2}, \ldots\right)$ - формальные переменные. Введем обозначение

$$
\mathscr{Q}=x_{2} \partial / \partial x_{1}+x_{3} \partial / \partial x_{2}+\ldots+y_{2} \partial / \partial y_{1}+y_{3} \partial / \partial y_{2}+\ldots
$$

Для композиции операторов $\mathscr{Q}$ и $\partial / \partial x_{i}$, а также $\mathscr{Q}$ и $\partial / \partial y_{i}$ имеют место следующие утверждения. 
Лемма 1. Для каждого $i \geqslant 2$ справедливо соотношение

$$
\frac{\partial}{\partial x_{i}} \mathscr{Q}=\mathscr{Q} \frac{\partial}{\partial x_{i}}+\frac{\partial}{\partial x_{i-1}}
$$

и, кроме того,

$$
\frac{\partial}{\partial x_{1}} \mathscr{Q}=\mathscr{Q} \frac{\partial}{\partial x_{1}}
$$

Доказателъство. Несложными преобразованиями левой части равенства (3) получаем его правую часть:

$$
\begin{aligned}
\frac{\partial}{\partial x_{i}} \mathscr{Q} & =\frac{\partial}{\partial x_{i}}\left(\sum_{j=1}^{\infty}\left(x_{j+1} \frac{\partial}{\partial x_{j}}+y_{j+1} \frac{\partial}{\partial y_{j}}\right)\right) \\
& =\sum_{j \neq i-1}\left(x_{j+1} \frac{\partial^{2}}{\partial x_{i} \partial x_{j}}+y_{j+1} \frac{\partial^{2}}{\partial y_{i} \partial y_{j}}\right)+\frac{\partial}{\partial x_{i-1}}+x_{i} \frac{\partial^{2}}{\partial x_{i} \partial x_{i-1}}+y_{i} \frac{\partial^{2}}{\partial y_{i} \partial y_{i-1}} \\
& =\sum_{j=1}^{\infty}\left(x_{j+1} \frac{\partial^{2}}{\partial x_{j} \partial x_{i}}+y_{j+1} \frac{\partial^{2}}{\partial y_{j} \partial y_{i}}\right)+\frac{\partial}{\partial x_{i-1}}=\mathscr{Q} \frac{\partial}{\partial x_{i}}+\frac{\partial}{\partial x_{i-1}} .
\end{aligned}
$$

Подобным же образом можно убедиться и в справедливости соотношения (4). Лемма доказана.

Отметим, что в частном случае $y_{i}=0$ соотношения (3) и (4) получены в [3].

Аналогично может быть доказана следующая лемма.

Лемма 2. Для каждого $i \geqslant 2$ справедливо соотношение

$$
\frac{\partial}{\partial y_{i}} \mathscr{Q}=\mathscr{Q} \frac{\partial}{\partial y_{i}}+\frac{\partial}{\partial y_{i-1}}
$$

u, кроме того,

$$
\frac{\partial}{\partial y_{1}} \mathscr{Q}=\mathscr{Q} \frac{\partial}{\partial y_{1}}
$$

Теорема 1. Полиномъ Туиара удовлетворяют системе дифференциально-разностнъх уравнений

$$
T_{n k}(x, y)=x_{1} T_{n-1, k-1}(x, y)+y_{1} T_{n-1, k}(x, y)+\mathscr{Q} T_{n-1, k}(x, y),
$$

2de $n, k \geqslant 1, n \geqslant k-1$.

Дохазательство. Известно (см. [5]), что

$$
T_{n, k}(x, y)=\sum_{j=0}^{n}\left(\begin{array}{l}
n \\
j
\end{array}\right) A_{j, k}(x) Y_{n-j}(y)
$$

Используя известное соотношение для биномиальных коэффициентов, последнее равенство перепишем в виде

$$
T_{n, k}(x, y)=\sum_{j=k}^{n}\left(\begin{array}{c}
n-1 \\
j-1
\end{array}\right) A_{j, k}(x) Y_{n-j}(y)+\sum_{j=k}^{n}\left(\begin{array}{c}
n-1 \\
j
\end{array}\right) A_{j, k}(x) Y_{n-j}(y)
$$


Рассмотрим первую сумму в правой части (9). Учитывая соотношение

$$
A_{n+1, k}(x)=x_{1} A_{n, k-1}(x)+\mathscr{D} A_{n, k}(x)
$$

(см. [2]), где

$$
\mathscr{D}=x_{2} \partial / \partial x_{1}+x_{3} \partial / \partial x_{2}+\ldots,
$$

находим, что

$$
\begin{aligned}
\sum_{j=k}^{n}\left(\begin{array}{c}
n-1 \\
j-1
\end{array}\right) A_{j, k}(x) Y_{n-j}(y)= & \sum_{j=k}^{n}\left(\begin{array}{c}
n-1 \\
j-1
\end{array}\right)\left(x_{1} A_{j-1, k-1}(x)+\mathscr{D} A_{j-1, k}(x)\right) Y_{n-j}(y) \\
= & x_{1} \sum_{j=k}^{n}\left(\begin{array}{c}
n-1 \\
j-1
\end{array}\right) A_{j-1, k-1}(x) Y_{n-j}(y) \\
& +\mathscr{D} \sum_{j=k}^{n}\left(\begin{array}{c}
n-1 \\
j-1
\end{array}\right) A_{j-1, k}(x) Y_{n-j}(y) \\
= & x_{1} \sum_{j=k-1}^{n-1}\left(\begin{array}{c}
n-1 \\
j
\end{array}\right) A_{j, k-1}(x) Y_{n-1-j}(y) \\
& +\mathscr{D} \sum_{j=k}^{n-1}\left(\begin{array}{c}
n-1 \\
j
\end{array}\right) A_{j, k}(x) Y_{n-1-j}(y) .
\end{aligned}
$$

Из последнего равенства и соотношения (8) получаем, что

$$
\sum_{j=k}^{n}\left(\begin{array}{c}
n-1 \\
j-1
\end{array}\right) A_{j, k}(x) Y_{n-j}(y)=x_{1} T_{n-1, k-1}(x, y)+\mathscr{D} T_{n-1, k}(x, y)
$$

Рассмотрим вторую сумму в правой части (9). Учитывая соотношение

$$
Y_{n+1}(y)=\left(y_{1}+\mathscr{P}\right) Y_{n}(y)
$$

(см. [2]), где

$$
\mathscr{P}=y_{2} \partial / \partial y_{1}+y_{3} \partial / \partial y_{2}+\ldots
$$

получаем, что

$$
\begin{aligned}
\sum_{j=k}^{n}\left(\begin{array}{c}
n-1 \\
j
\end{array}\right) A_{j, k}(x)\left(y_{1}+\mathscr{P}\right) Y_{n-j-1}(y)= & y_{1} \sum_{j=k}^{n}\left(\begin{array}{c}
n-1 \\
j
\end{array}\right) A_{j, k}(x) Y_{n-1-j}(y) \\
& +\mathscr{P} \sum_{j=k}^{n}\left(\begin{array}{c}
n-1 \\
j
\end{array}\right) A_{j, k}(x) Y_{n-1-j}(y) \\
= & y_{1} \sum_{j=k}^{n-1}\left(\begin{array}{c}
n-1 \\
j
\end{array}\right) A_{j, k}(x) Y_{n-1-j}(y) \\
& +\mathscr{P} \sum_{j=k}^{n-1}\left(\begin{array}{c}
n-1 \\
j
\end{array}\right) A_{j, k}(x) Y_{n-1-j}(y) .
\end{aligned}
$$


Из последнего равенства и соотношения (8) находим, что

$$
\sum_{j=k}^{n}\left(\begin{array}{c}
n-1 \\
j
\end{array}\right) A_{j, k}(x) Y_{n-j}(y)=y_{1} T_{n-1, k}(x, y)+\mathscr{P} T_{n-1, k}(x, y)
$$

Подставив соотношения (10) и (11) в (9) и учитывая, что $\mathscr{Q}=\mathscr{D}+\mathscr{P}$, получим соотношение (7). Теорема доказана.

Отметим, что в частном случае $y_{i}=0, \mathscr{Q}=\mathscr{D}$ соотношение (7) для частичных полиномов Белла приводится в [2].

Для полиномов $T_{n k}(x, y)$ имеют место следующие утверждения.

Теорема 2. Для $n \geqslant 1$ справедливы соотношения

$$
\frac{\partial}{\partial x_{i}} T_{n k}(x, y)=\left(\begin{array}{c}
n \\
i
\end{array}\right) T_{n-i, k-1}(x, y)
$$

гдe $k, i \geqslant 1, k+i \leqslant n+1$.

Доказателъство. Доказательство проведем индукцией по $n$ отдельно для $i=1$ и $i \geqslant 2$.

Докажем соотношение (12) при $i=1$. Для всех $n=k \geqslant 1$ и $n=2, k=1$ оно справедливо, поскольку

$$
T_{n n}(x, y)=x_{1}^{n}, \quad T_{21}(x, y)=2 x_{1} y_{1}+x_{2}, \quad T_{10}(x, y)=y_{1} .
$$

Пусть равенство (12) при $i=1$ выполняется для всех $n<m$, где $m$ - целое число, $m \geqslant 3$. От обеих частей соотношения (7), полагая в нем $n=m$, берем частные производные по $x_{1}$. Тогда

$$
\begin{aligned}
\frac{\partial}{\partial x_{1}} T_{m k}(x, y)= & \frac{\partial}{\partial x_{1}}\left(x_{1} T_{m-1, k-1}\right)(x, y) \\
& +\frac{\partial}{\partial x_{1}}\left(y_{1} T_{m-1, k}\right)(x, y)+\frac{\partial}{\partial x_{1}}\left(\mathscr{Q} T_{m-1, k}(x, y)\right)
\end{aligned}
$$

Согласно формуле (4) и предположению индукции из последнего соотношения получим, что

$$
\begin{aligned}
\frac{\partial}{\partial x_{1}} T_{m k}(x, y)= & T_{m-1, k-1}(x, y)+x_{1} \frac{\partial}{\partial x_{1}} T_{m-1, k-1}(x, y) \\
& \quad+y_{1} \frac{\partial}{\partial x_{1}} T_{m-1, k}(x, y)+\mathscr{Q} \frac{\partial}{\partial x_{1}} T_{m-1, k}(x, y) \\
= & T_{m-1, k-1}(x, y)+x_{1}(m-1) T_{m-2, k-2}(x, y) \\
& \quad+y_{1}(m-1) T_{m-2, k-1}(x, y)+\mathscr{Q}\left((m-1) T_{m-2, k-1}(x, y)\right. \\
= & T_{m-1, k-1}(x, y)+(m-1)\left(x_{1} T_{m-2, k-2}(x, y)\right. \\
& \left.\quad+y_{1} T_{m-2, k-1}(x, y)+\mathscr{Q} T_{m-2, k-1}(x, y)\right) \\
= & T_{m-1, k-1}(x, y)+(m-1) T_{m-1, k-1}(x, y) \\
= & m T_{m-1, k-1}(x, y),
\end{aligned}
$$


что и дает соотношение (12) при $i-1, n=m$.

Докажем соотношение (12) при $2 \leqslant i \leqslant n-k+1$. Для $i=2, n=2, k=1$ оно проверяется непосредственно, поскольку

$$
T_{21}(x, y)=2 x_{1} y_{1}+x_{2}, T_{00}(x, y)=1 \text {. }
$$

Пусть равенство (12) при $2 \leqslant i \leqslant n-k+1$ справедливо для всех $n<m$ (а, следовательно, и для $i<m), m>3$. Взяв от обеих частей соотношения (7) при $n=m$ частные производные по $x_{i}, 2 \leqslant i \leqslant m-1$, получим, что

$$
\begin{aligned}
\frac{\partial}{\partial x_{i}} T_{m k}(x, y)= & \frac{\partial}{\partial x_{i}}\left(x_{1} T_{m-1, k-1}(x, y)\right) \\
& \quad+\frac{\partial}{\partial x_{i}}\left(y_{1} T_{m-1, k}(x, y)\right)+\frac{\partial}{\partial x_{i}}\left(\mathscr{Q} T_{m-1, k}(x, y)\right) .
\end{aligned}
$$

Из последнего соотношения согласно равенствам (3), (7) (при $i=2$ ) и формуле (12) уже доказанной для $i=1$ по предположению индукции

$$
\begin{aligned}
\frac{\partial}{\partial x_{i}} T_{m k}(x, y)= & x_{1} \frac{\partial}{\partial x_{i}} T_{m-1, k-1}(x, y)+y_{1} \frac{\partial}{\partial x_{i}} T_{m-1, k} \\
& +\mathscr{Q} \frac{\partial}{\partial x_{i}} T_{m-1, k}(x, y)+\frac{\partial}{\partial x_{i-1}} T_{m k}(x, y) \\
= & x_{1}\left(\begin{array}{c}
m-1 \\
i
\end{array}\right) T_{m-i-1, k-2}(x, y)+y_{1}\left(\begin{array}{c}
m-1 \\
i
\end{array}\right) T_{m-i-1, k-1}(x, y) \\
& \quad+\mathscr{Q}\left(\begin{array}{c}
m-1 \\
i
\end{array}\right) T_{m-i-1, k-1}(x, y)+\left(\begin{array}{c}
m-1 \\
i
\end{array}\right) T_{m-i, k-1}(x, y) \\
= & \left(\begin{array}{c}
m-1 \\
i
\end{array}\right)\left(x_{1} T_{m-i-1, k-2}(x, y)+y_{1} T_{m-i-1, k-1}(x, y)\right. \\
& \left.+\mathscr{Q} T_{m-i-1, k-1}(x, y)\right)+\left(\begin{array}{c}
m-1 \\
i-1
\end{array}\right) T_{m-i, k-1}(x, y) \\
= & \left(\begin{array}{c}
m-1 \\
i
\end{array}\right) T_{m-i, k-1}(x, y)+\left(\begin{array}{c}
m-1 \\
i-1
\end{array}\right) T_{m-i, k-1}(x, y) \\
= & \left(\begin{array}{c}
m \\
i
\end{array}\right) T_{m-i, k-1}(x, y) .
\end{aligned}
$$

Сопоставление первого и последнего звеньев полученной цепочки равенств дает соотношение (12) для $2 \leqslant i \leqslant n-k, n=m$.

Убедимся в справедливости равенства (12) при $i=m$. Пусть равенство (12) справедливо при $i=n=m-1$. Взяв от обеих частей соотношения (7) при $n=m$ частные производные по $x_{m}$, получим равенство

$$
\begin{aligned}
\frac{\partial}{\partial x_{m}} T_{m k}(x, y)= & \frac{\partial}{\partial x_{m}}\left(x_{1} T_{m-1, k-1}(x, y)\right) \\
& \quad+\frac{\partial}{\partial x_{m}}\left(y_{1} T_{m-1, k}(x, y)\right)+\frac{\partial}{\partial x_{m}}\left(\mathscr{Q} T_{m-1, k}(x, y)\right) .
\end{aligned}
$$

Поскольку, согласно формуле (1), $\partial / \partial x_{m} T_{m k}(x, y) \neq 0$ лишь при $k=1$ и, кроме того, $\partial / \partial x_{m} T_{m-1, k}(x, y)=0$, предыдущее соотношение примет вид

$$
\frac{\partial}{\partial x_{m}} T_{m 1}(x, y)=\frac{\partial}{\partial x_{m}}\left(\mathscr{Q} T_{m-1,1}(x, y)\right) \text {. }
$$


Отсюда, согласно равенству (3) и предположению индукции, находим, что

$$
\begin{aligned}
\frac{\partial}{\partial x_{m}} T_{m 1}(x, y) & =\mathscr{Q}\left(\frac{\partial}{\partial x_{m}} T_{m-1,1}(x, y)\right)+\frac{\partial}{\partial x_{m-1}} T_{m-1,1}(x, y) \\
& =\left(\begin{array}{l}
m-1 \\
m-1
\end{array}\right) T_{0,0}(x, y),
\end{aligned}
$$

что приводит к равенству

$$
\frac{\partial}{\partial x_{m}} T_{m 1}(x, y)=T_{0,0}(x, y)
$$

то есть соотношению (12) при $i=n=m$. Теорема доказана.

Отметим, что в случае $y_{i}=0$ из формулы (12) получаем соотношение для частичных полиномов Белла, приведенное, например, в [9].

Также методом математической индукции с использованием формулы (7) и соотношений (5) и (6) может быть доказана следующая теорема.

Теорема 3. Для $n \geqslant 1$ справедливы соотношения

$$
\frac{\partial}{\partial y_{i}} T_{n k}(x, y)=\left(\begin{array}{c}
n \\
i
\end{array}\right) T_{n-i, k}(x, y)
$$

где $k \geqslant 0, i \geqslant 1, k+i \leqslant n$.

В [6] рассматривается следующий частный случай полиномов Тушара:

$$
T_{n, k}^{*}(x, y)=T_{n, k}\left(x_{1}^{*}, \ldots, x_{n}^{*} ; y_{1}^{*}, \ldots, y_{n}^{*}\right),
$$

где $x_{k}^{*}=(k-1) ! x_{k}, y_{k}^{*}=(k-1) ! y_{k}, k=1, \ldots, n$. Очевидно, что для полиномов $T_{n, k}^{*}(x, y)$ справедливы все утверждения теорем $1-3$.

\section{4. Рекуррентные соотношения для полиномов}

Широко известные цикловые индикаторы симметрической группы имеют следующий вид

$$
C_{n}(t)=C_{n}\left(t_{1}, \ldots, t_{n}\right)=\sum \frac{n !}{k_{1} ! \ldots k_{n} !}\left(\frac{t_{1}}{1}\right)^{k_{1}} \ldots\left(\frac{t_{n}}{n}\right)^{k_{n}},
$$

(см., например, [2]), где $t=\left(t_{1}, \ldots, t_{n}\right)$ - формальные переменные, а суммирование ведется по всем таким наборам $\left(k_{1}, \ldots k_{n}\right)$ целых неотрицательных чисел, что

$$
k_{1}+2 k_{2}+\ldots+n k_{n}=n \text {. }
$$

Дополнительно полагают, что $C_{0}=1$.

Цикловые индикаторы связаны с полиномами Белла соотношениями

$$
C_{n}(t)=Y_{n}\left(t_{1}, t_{2}, 2 ! t_{3}, \ldots,(n-1) ! t_{n}\right)
$$

(см., например, [2]). 
В [6] при помощи соотношения

$$
C_{n, k}(x, y)=C_{n, k}\left(x_{1}, \ldots ; y_{1}, \ldots\right)=\left(\begin{array}{l}
n \\
k
\end{array}\right) C_{k}(x) C_{n-k}(y)
$$

где $0 \leqslant k \leqslant n, n \geqslant 1$, введены полиномы Тушара $C_{n, k}(x, y)$. Просуммируем по $k$ обе части равенства (14):

$$
\sum_{k=0}^{n} C_{n, k}(x, y)=\sum_{k=0}^{n}\left(\begin{array}{l}
n \\
k
\end{array}\right) C_{k}(x) C_{n-k}(y)
$$

Далее, перейдя по формуле (13) к полиномам Белла, получим, что

$$
\begin{aligned}
& \sum_{k=0}^{n} C_{n, k}(x, y) \\
& \quad=\sum_{k=0}^{n}\left(\begin{array}{l}
n \\
k
\end{array}\right) Y_{k}\left(x_{1}, x_{2}, 2 ! x_{3}, \ldots,(k-1) ! x_{k}\right) Y_{n-k}\left(y_{1}, y_{2}, 2 ! y_{3}, \ldots,(n-k-1) ! y_{n-k}\right) .
\end{aligned}
$$

Сопоставляя полученное выражение с формулой (2), находим, что полиномы Тушара $T_{n, k}^{*}(x, y)$ и $C_{n, k}(x, y)$ связаны соотношением

$$
\sum_{k=0}^{n} C_{n, k}(x, y)=\sum_{k=0}^{n} T_{n, k}\left(x^{*}, y^{*}\right)
$$

где $x_{i}^{*}=(i-1) ! x_{i}, y_{i}^{*}=(i-1) ! y_{i}, i=1, \ldots, n$.

Теорема 4. Полиномы $C_{n, k}(x, y)$ удовлетворяют рехуррентному соотношению

$$
C_{n+1, k}(x, y)=\sum_{i=0}^{k-1}(n)_{i} x_{i+1} C_{n-i, k-i-1}(x, y)+\sum_{i=0}^{n-k}(n)_{i} y_{i+1} C_{n-i, k}(x, y)
$$

Дохазательство. В книге [2] приводится рекуррентная формула для цикловых индикаторов симметрических групп

$$
C_{n+1}(t)=\sum_{i=0}^{n}(n)_{i} t_{i+1} C_{n-i}(t)
$$

Полагая в формуле (16) $t_{i}=x_{i}, n=k-1$, получаем, что

$$
C_{k}(x)=\sum_{i=0}^{k-1}(k-1)_{i} x_{i+1} C_{k-i-1}(x)
$$

Подставляя последнее соотношение в (14), находим, что

$$
\begin{aligned}
C_{n+1, k}(x, y) & =\left(\begin{array}{c}
n+1 \\
n
\end{array}\right) C_{n-k-1}(y) \sum_{i=0}^{k-1}(k-1)_{i} x_{i+1} C_{k-i+1}(x) \\
& =\frac{n+1}{k} \sum_{i=0}^{k-1}(n)_{i} x_{i+1} C_{n-i, k-i-1}(x, y)
\end{aligned}
$$


или

$$
\frac{k}{n+1} C_{n+1, k}(x, y)=\sum_{i=0}^{k-1}(n)_{i} x_{i+1} C_{n-i, k-i-1}(x, y) .
$$

Аналогично из формулы (16) при $t_{i}=y_{i}, n=n-k$ получаем равенство

$$
C_{n-k+1}(y)=\sum_{i=0}^{n-k}(n-k)_{i} y_{i+1} C_{n-k-i}(y) \text {. }
$$

Подставляя данное соотношение в (14), находим, что

$$
\begin{aligned}
C_{n+1, k}(x, y) & =\left(\begin{array}{c}
n+1 \\
k
\end{array}\right) C_{k}(x) \sum_{i=0}^{n-k}(n-k)_{i} y_{i+1} C_{n-k-i}(y) \\
& =\frac{n+1}{n+1-k} \sum_{i=0}^{n-k}(n)_{i} y_{i+1} C_{n-i, k}(x, y)
\end{aligned}
$$

Отсюда следует, что

$$
\frac{n+1-k}{n+1} C_{n+1, k}(x, y)=\sum_{i=0}^{n-k}(n)_{i} y_{i+1} C_{n-i, k}(x, y)
$$

Суммируя соответственно правые и левые части равенств (17) и (18), получим соотношение (15). Теорема доказана.

\section{5. Перечислительные интерпретации}

Обсудим некоторые перечислительные интерпретации изучаемых комбинаторных объектов.

Для полиномов Тушара $T_{n, k}(x, y)$ известна следующая перечислительная интерпретация (см. [5]). Число $T_{n k}(x, y)$ равно числу перестановок $n$ элементов, скажем, $u_{1}, \ldots, u_{n}$, в которых точно $k$ циклов обладают свойством $A$, а все остальные свойством $B$, где $x_{j}, 1 \leqslant j \leqslant n$, есть число циклических перестановок с циклом длины $j$, которые обладают свойством $A$, а $y_{j}, 1 \leqslant j \leqslant n$, есть число циклических перестановок с циклом длины $j$, которые обладают свойством $B$, при условии, что каждый цикл длины $j$ определяет одно из чисел $x_{j}$ или $y_{j}$.

В [6] приводится один специальный случай указанной комбинаторной интерпретации.

Пусть свойство $A$ задано следующим образом: элементы $u_{i_{1}}, \ldots, u_{i_{j}}$ цикла длины $j, 1 \leqslant j \leqslant n$, появляются так, что $i_{1}<i_{2}<\ldots<i_{j}$, а свойство $B$ состоит в том, что элементы $u_{i_{1}}, \ldots, u_{i_{j}}$ цикла длины $j, 1 \leqslant j \leqslant n$, появляются на любых местах, исключая те, которые соответствуют свойству $A$. Тогда $x_{j}=1,1 \leqslant j \leqslant n$, и $y_{j}=(j-1) !-1,1 \leqslant j \leqslant n$, и число перестановок из $n$ элементов, в которых точно $k$ циклов, обладающих свойством $A$, равно

$$
T_{n k}(1,1, \ldots ; 0,0,1, \ldots,(j-1) !-1, \ldots)=\sum_{i=0}^{n}(n)_{i} \sum_{j=0}^{n-k-i}(-1)^{j}\left(\begin{array}{c}
k+j \\
k
\end{array}\right) S(n-i, k+j)
$$


где $S(n, k)$ - числа Стирлинга второго рода.

Можно предложить следующую перечислительную интерпретацию полиномов Тушара $T_{n, k}(x, y)$. Рассмотрим все разбиения $n$-множества $N_{n}$ на непустые блоки, среди которых $k$ блоков обладают свойством $A$, а остальные - свойством $B$. Вес блока, состоящего из $j$ элементов, обладающих свойством $A$, считаем равным $x_{j}$, вес элемента со свойством $B$ - равным $y_{j}$, а вес всего разбиения - равным произведению весов его блоков. Тогда $T_{n, k}(x, y)$ есть сумма весов всех разбиений $N_{n}$ на непустые блоки, среди которых $k$ блоков обладают свойством $A$, а остальные - свойством $B$.

Из приведенных интерпретаций при $y_{j}=0$ получаем известные интерпретации частичных полиномов Белла $A_{n, k}(x)$ (см., например, $[10,11]$ и [3]).

Полиномам $T_{n, k}^{*}(x, y)$ можно дать следующую перечислительную интерпретацию. Число $T_{n, k}^{*}(x, y)$ равно сумме весов всех подстановок степени $n$, где $k$ циклов обладают свойством $A$, а все остальные - свойством $B$, при условии, что вес цикла длины $j$, который обладает свойством $A$ равен $x_{j}^{*}=(j-1) ! x_{j}$, вес цикла со свойством $B$ равен $y_{j}^{*}=(j-1) ! y_{j}$, а вес подстановки равен произведению весов составляющих ее циклов.

Из полученной интерпретации при $y_{j}^{*}=0$ получаем перечислительную интерпретацию полиномов $C_{n}(x)$, а при $x_{j}^{*}=1$ и $y_{j}^{*}=0$ - интерпретацию чисел Стирлинга 1 рода $s(n, k)$ (см., например, [2]).

\section{6. Применения}

Рассмотрим одноканальную систему массового обслуживания с ожиданием (см., например, [12]). Пусть $t_{0}=0, t_{1}, t_{2}, \ldots$ - моменты поступлений требований и $v_{1}, v_{2}, \ldots$ - времена их обслуживания. Тогда $u_{n}=t_{n}-t_{n-1}, n \geqslant 1$, представляют собой интервалы между поступлениями требований в систему. Положим

$$
\zeta_{k}=v_{k-1}-u_{k}, \quad k \geqslant 1
$$

и

$$
S_{0} \equiv 0, \quad S_{n}=\zeta_{1}+\zeta_{2}+\ldots+\zeta_{n}, \quad n \geqslant 1 .
$$

Будем считать, что $\zeta_{k}, k \geqslant 1$, - взаимно независимые одинаково распределенные случайные величины и

$$
F(x)=\mathbf{P}\left\{\zeta_{i} \leqslant x\right\}, \quad-\infty<x<\infty, \quad 1 \leqslant i \leqslant n .
$$

Последовательность $\left\{S_{n}, n \geqslant 0\right\}$ представляет собой случайное блуждание, соответствующее функции распределения $F(x)$. Для него определим две последовательности случайных величин $\left\{N_{k}, k \geqslant 0\right\}$ и $\left\{\bar{N}_{k}, k \geqslant 0\right\}$, полагая

$$
\begin{aligned}
& \bar{N}_{0} \equiv 0, \quad \bar{N}_{1}=\min \left\{n>0: S_{n} \leqslant 0\right\}, \quad \bar{N}_{k}=\min \left\{n>\bar{N}_{k-1}: S_{n} \leqslant S_{\bar{N}_{k-1}}\right\}, \quad k \geqslant 2 ; \\
& N_{0} \equiv 0, \quad N_{1}=\min \left\{n: S_{n}>0\right\}, \quad N_{k}=\min \left\{n: S_{n}>S_{N_{k}-1}\right\}, \quad k \geqslant 2 .
\end{aligned}
$$

Случайная величина $N_{k}$ называется $k$-м верхним лестничным моментом, а $S_{N_{k}}$ соответственно $k$-й лестничной высотой. Аналогично, $\bar{N}_{k}$ и $S_{\bar{N}_{k}}-$ нижние $k$-е лестничные момент и высота. Пары случайных величин $\left(N_{k}, S_{N_{k}}\right)$ и $\left(\bar{N}_{k}, S_{\bar{N}_{k}}\right)$ назовем 
соответственно $k$-й верхней и $k$-й нижней лестничной точкой случайного блуждания $S_{n}$. Для удобства положим $N_{1}=N, \bar{N}_{1}=\bar{N}$. Совместное распределение первой верхней лестничной точки $\left(N, S_{N}\right)$ можно записать в виде

$$
\begin{aligned}
f_{n}(x) & =\mathbf{P}\left\{N=n, S_{N} \leqslant x\right\} \\
& =\mathbf{P}\left\{S_{1} \leqslant 0, S_{2} \leqslant 0, \ldots, S_{n-1} \leqslant 0,0<S_{n} \leqslant x\right\} .
\end{aligned}
$$

Аналогично совместное распределение первой нижней лестничной точки может быть записано в виде

$$
\begin{aligned}
g_{n}(x) & =\mathbf{P}\left\{\bar{N}=n, S_{\bar{N}} \leqslant x\right\} \\
& =\mathbf{P}\left\{S_{1}>0, S_{2}>0, \ldots, S_{n-1}>0, S_{N} \leqslant x<0\right\} .
\end{aligned}
$$

Найдем данные вероятности, используя полиномы Тушара. Пусть $N_{n}$ - число первых положительных частичных сумм $S_{k}, 1 \leqslant k \leqslant n$. Известно, (см., например, [13]), чTо

$$
\mathbf{P}\left\{N_{n}=n\right\}=\mathbf{P}\left\{\nu_{1} \delta\left(S_{\nu_{1}}\right)+\ldots+\nu_{r} \delta\left(S_{\nu_{r}}\right)=n\right\},
$$

где $\nu_{1}, \ldots, \nu_{r}$ - случайные величины, характеризующие длину непересекающихся циклов перестановки, выбранной случайным образом среди перестановок $n$ объектов, причем $\nu_{1}+\ldots+\nu_{r}=n, S_{\nu_{r}}=\zeta_{k_{\nu_{1}}}+\ldots+\zeta_{k_{\nu_{r}}}$ и

$$
\delta\left(S_{\nu_{r}}\right)= \begin{cases}1, & \text { если } S_{\nu_{r}}>0 \\ 0, & \text { если } S_{\nu_{r}} \leqslant 0 .\end{cases}
$$

Отсюда следует, что мы ищем число перестановок, в которых все циклы обладают свойством $A$, состоящим в том, что сумма случайных величин, которые составляют цикл, положительна. Тушар [4] доказал, что число перестановок $n$ элементов, в которых все циклы обладают свойством $A$ выражается через

$$
A_{n}=Y_{n}\left(a_{1}^{*}, \ldots, a_{n}^{*}\right)
$$

где $a_{k}^{*}=(k-1) ! a_{k}, 1 \leqslant k \leqslant n$, так что

$$
g_{k}(x)=\frac{1}{k !} Y_{n}\left(a_{1}^{*}, \ldots, a_{n}^{*}\right)=\frac{1}{k !} C_{k}\left(a_{1}, \ldots, a_{k}\right) .
$$

Аналогично показывается, что

$$
f_{n-k}(x)=\frac{1}{(n-k) !} C_{n-k}\left(1-a_{1}, \ldots, 1-a_{n-k}\right),
$$

где $a_{k}=\mathbf{P}\left\{S_{k}>0\right\}$. Теперь можно найти вероятность того, что в очереди будет находиться $k-1$ требование:

$$
P_{k}(n)=\mathbf{P}\left\{N_{n}=k\right\}=\mathbf{P}\left\{S_{1}>0, S_{2}>0, \ldots, S_{k}>0, S_{k+1} \leqslant 0, S_{k+2} \leqslant 0, \ldots, S_{n} \leqslant 0\right\} .
$$

С учетом формулы (14) получаем искомую вероятность

$$
P_{k}(n)=\frac{1}{n !} C_{n, k}\left(a_{1}, \ldots, a_{n}, 1-a_{1}, \ldots, 1-a_{n}\right) .
$$

Из последнего соотношения с учетом (15) находим, что

$$
P_{k}(n+1)=\frac{1}{n+1}\left(\sum_{i=0}^{k-1} a_{i+1} P_{k-i-1}(n-i)+\sum_{i=0}^{n-k}\left(1-a_{i+1}\right) P_{k}(n-i)\right),
$$

где $a_{k}=\mathbf{P}\left\{S_{k}>0\right\}$. 


\section{Список литературы}

1. Bell E. T., Partition polynomials. Ann. Math. (1927) 29, 38-46.

2. Риордан Дж., Введение в комбияаторный анализ. ИЛ, Москва, 1963.

3. Кузьмин О. В., Рекуррентные соотношения и перечислительные интерпретации некоторых комбинаторных чисел и полиномов. Дискретная математиха (1994) 6, №3, 39-49.

4. Touchard J., Sur les cycles des substitutions. Acta Math. (1939) 70, №3-4, 243-297.

5. Chrysaphinou O., On Touchard polynomials. Discrete Math. (1985) 54, 143-152.

6. Charalambides Ch. A., Chrysaphinou O., Partition polynomials in fluctuation theory. Math. Nachr. (1982) 106, 89-100.

7. Кузьмин О. В., Леонова О. В., О полиномах Тушара. Асимптотические и перечислительнье задачи комбинаторного анализа. Иркут. ун-т, Иркутск, 1997, 101-109.

8. Comtet L., Advanced Combinatorics. Reidel, Dordrecht, 1974.

9. Селиванов Б. И., Комбинаторный подход к формуле обращения Бюрмана-Лагранжа. Комбинаторный и асимптотический анализ. Красноярский ун-т, Красноярск, 1977, 153-169.

10. Howard F. T., Bell polynomials and degenerate Stirling numbers. Rend. Sem. Mat. Univ. Padova (1980) 61, 203-219.

11. Frucht R. W., Rota G. C., Polynomios de Bell y partitiones de conjuntos finitos. Scientia (1965) 32, N2126, 5-10.

12. Гнеденко Б. В., Коваленко И. Н., Введение в теорию массового обслуживаяия. Наука, Москва, 1987.

13. Spitzer F., A combinatorial lemma and its applications to probability theory. Trans. Amer. Math. Soc. (1956) 82, 323-339.

Статья поступила 15.12.1998. 